\title{
A PRÁTICA EDUCATIVA NA PREVENÇÃO DE CONDIÇÕES CRÔNICAS
}

\author{
Bárbara Ferreira dos Santos \\ Pontifícia Universidade Católica de Minas Gerais \\ barbara_fs@hotmail.com.br \\ Aline Pires Ribeiro Salvador \\ Pontifícia Universidade Católica de Minas Gerais \\ aline27_ribeiro@yahoo.com.br \\ Livia Muniz Cirino de Carvalho \\ Pontifícia Universidade Católica de Minas Gerais \\ liviamunizc@hotmail.com
}

\author{
Carolina Gonçalves Hubner \\ Pontifícia Universidade Católica de Minas Gerais \\ carolina.hubnerx3@gmail.com \\ Natália de Cássia Horta \\ Pontifícia Universidade Católica de Minas Gerais \\ nanahorta@yahoo.com.br \\ Sabrina Alves Ramos \\ Pontifícia Universidade Católica de Minas Gerais \\ sabrinaalvesramos@gmail.com
}

\section{Resumo}

O artigo relata experiências vivenciadas por acadêmicos e docentes de graduação da área da saúde junto à comunidade em projeto de extensão desenvolvido de março a dezembro de 2015. Nesse artigo abordaram-se as práticas sobre Diabetes Mellitus, Hipertensão Arterial, Doenças Cardiovasculares e Obesidade, com orientações e demonstrações para a população em escola, restaurante e na universidade. As intervenções foram iniciadas com conversa com os participantes sobre meios de prevenção, redução de agravos, receitas saudáveis e modificações nos hábitos de vida. Todas as atividades de extensão abordaram formas de minimizar as doenças por meio da alimentação, com a oferta de preparações e receitas ou dinâmicas. As práticas revelaram interesse do público na mudança dos estilos de vida e trouxeram para os extensionistas conhecimentos, pois estes puderam colocar em prática conteúdos aprendidos em sala de aula, o que é um dos objetivos da extensão universitária.

Palavras-chave: Promoção da Saúde. Doença Crônica. Qualidade de Vida. Hábitos Alimentares.

\section{PRACTICE EDUCATION IN THE CHRONICLES CONDITIONS PREVENTION}

Abstract

This article reports experiences of undergraduates and university teatchers with the community in an extension project developed from March to December of 2015. In this article, the practices of Diabetes Mellitus, Hypertension, Cardiovascular Diseases and Obesity were discussed, with guidelines and demonstrations for the population at school, restaurant and university. The interventions were initiated by talking with the participants about means of prevention, reduction of diseases, healthy recipes and modifications in the habits of life. Practices to combat hypertension and diabetes have addressed ways to minimize diseases through eating and the ones to combat obesity and cardiovascular diseases were performed through dynamics. The practices revealed public interest in changing lifestyles and brought to extensionists practical knowledge, because they were able to put into practice content learned in class, which is one of the objectives of university extension.

Keywords: Health Promotion. Chronic Diseases. Quality of Life. Food Habits.

\section{LA PRATICA EDUCATIVA EN LA PREVENCION DE CONDICIONES CRONICAS} Resumen

Este artículo trata de las experiencias vividas por estudiantes y docentes de pregrado con la comunidad en un proyecto de extensión universitaria desarrollado entre marzo y diciembre de 2015. En este artículo son abordadas prácticas sobre Diabetes Mellitus, Hipertensión Arterial, Enfermedad Cardiovascular y Obesidad, con orientación y demostraciones para la población en escuela restaurante y en la universidad. Las intervenciones empezaron por una conversación acerca de las formas de prevenir, reducir agravamientos, discusión de recetas saludables y cambio en los hábitos de vida. Las prácticas de combate a la Hipertensión Arterial y al Diabetes Mellitus abordaron medios de minimizar la enfermedad por medio de la alimentación. Las prácticas de combate a la obesidad y a las Enfermedades Cardiovasculares fueran hechas por medio de dinámicas. La práctica ha revelado interés de la población en el cambio de los hábitos alimenticios y trajeron a los extensionistas conocimientos prácticos, porque ellos pudieron poner en práctica los contenidos aprendidos en las aulas, o que es uno de los objetivos de la extensión universitaria.

Palabras clave: Promoción de la Salud. Enfermedades Crónicas. Calidad de Vida. Hábitos Alimenticios. 


\section{INTRODUÇÃO}

A extensão universitária faz parte da Constituição da República Federativa do Brasil de 1988, na qual se explicitou que as universidades deveriam conciliar a sua autonomia didáticocientífica, administrativa, de gestão financeira e patrimonial, para fazer com que a graduação pudesse se estabelecer em três pilares inseparáveis: ensino, pesquisa e extensão (DIVINO, 2013). Nesse contexto, o desenvolvimento de projetos de extensão é uma estratégia importante para a efetivação da sociabilidade, na qual articula ações de vigilância em saúde ao cotidiano dos indivíduos envolvidos na construção de ações sociais, bem como propicia a vivência acadêmica na interface com a comunidade nas suas necessidades. Trata-se de uma prática dual em que quem aprende também ensina, ou seja, tanto a comunidade quanto a universidade se beneficiam nessa integração.

De acordo com Oliveira (2004) a extensão proporciona aos acadêmicos cooperação para a formação dos currículos, alimentando e subsidiando a relação entre a Sociedade e Universidade, com o objetivo de reconstruir e ressignificar a história da Universidade diante da Sociedade. O convívio representa uma ferramenta enriquecedora, quando se deseja promover saúde em âmbito coletivo, atividades comunitárias são capazes de transformar simples tarefas acadêmicas em fortes laços sociais que contribuem efetivamente com a formação do indivíduo enquanto cidadão responsável por intervir nas melhorias e transformações da sociedade a qual está inserido. Ao introduzir uma proposta de atividade, da qual a sua execução exige o envolvimento de diversas pessoas e organizações, é proporcionado ao estudante o desenvolvimento de práticas e habilidades em grupo que são essenciais para o seu futuro profissional (SANTOS e CASTRO, 2014).

O Brasil passa por um período denominado de "transição nutricional", em que o perfil epidemiológico está diretamente relacionado às mudanças nos hábitos de vida da população. De acordo com a Organização Mundial de Saúde (OMS), por volta da década de 1940 havia no país um grande número de mortes por doenças infecciosas e, atualmente, são as doenças crônicas não transmissíveis (DCNT), como, por exemplo, doenças cardiovasculares, Diabetes Mellitus Tipo II, hipertensão arterial e obesidade que expressam o maior índice de morbimortalidade no país e no mundo (OMS, 2014). Além disso, estas doenças impactam diretamente na funcionalidade, produtividade e na qualidade de vida da população podendo resultar em altos custos no setor da saúde (MANSO et al., 2016). Essas patologias são hoje consideradas como um problema de saúde global, um risco gravíssimo à saúde e ao desenvolvimento humano, tidas como condições crônicas. Observa-se que as DCNT estão altamente ligadas aos hábitos de vida, além de também 
estarem relacionadas com gênero, idade, situação econômica, estado de saúde, entre outros (MELLO et al., 2016). A grande carga dessas doenças destaca-se principalmente em países em desenvolvimento, onde grande parte da população possui baixa e média renda (SCHMIDT et al., 2011).

Segundo dados da Pesquisa Nacional de Saúde (PNS) realizada entre agosto de 2013 a fevereiro de 2014 , cerca de $40 \%$ da população adulta brasileira, o equivalente a 57,4 milhões de pessoas, possuem pelo menos uma DCNT, sendo as mesmas responsáveis por mais de $72 \%$ das causas de mortes no Brasil (PORTAL BRASIL, 2014). As principais DCNT e seus fatores de risco compartilhados integram um plano de ação da OMS que tem por foco as doenças cardiovasculares, diabetes, câncer e doenças respiratórias crônicas (DUNCAN et al., 2012).

As DCNT são elencadas como prioridade no setor de Saúde Pública pela morbidade e mortalidade. Embora o crescimento tenha ocorrido rapidamente nos últimos anos, seu impacto é passível de reverter ou frear, por meio de medidas preventivas e custo-efetivas, rastreio e detecção precoce (MALTA; MORAIS NETO; SILVA JÚNIOR, 2011). Campanhas de prevenção têm possibilitado redução de mortalidade por doenças cardiovasculares em algumas regiões brasileiras (VILLELA; GOMES; MELÉNDEZ, 2014). Desta forma, o desenvolvimento de ações no âmbito de promoção da saúde, incluindo o autocuidado apoiado à gestão de caso nas situações de maior complexidade de saúde, devem ser realizadas (MENDES, 2012). CORRÊA et al. (2015, p. 15) afirma que "a promoção em saúde tem como objetivos modificar a visão assistencialista da saúde por meio da formulação e implementação de políticas saudáveis, movimentos organizados e iniciativas em programas nos diversos setores da sociedade".

Deste modo, ter maior compreensão das DCNT presentes na sociedade e buscar meios para preveni-las é primordial para sustentar o bem-estar e a boa qualidade de vida da população, uma vez que esses fatores são determinantes para prevenção, controle e tratamento de tais doenças que, em longo prazo, podem trazer graves problemas de saúde. Atrelado a isso, o reconhecimento das condições de vida e o estímulo aos hábitos saudáveis - tidos como os modos de vida - devem nortear as ações cuidadoras em saúde com a centralidade nos determinantes sociais.

Neste artigo, buscou-se relatar experiências de práticas educativas preventivas de doenças crônicas, com foco em doenças cardiovasculares, Diabetes Mellitus, hipertensão arterial e obesidade, frente à importância da educação nutricional para prevenir agravos advindos dessas patologias. 


\section{MATERIAIS E MÉTODOS}

As práticas extensionistas são oriundas do Projeto "Retratos do Cotidiano em Saúde: A Vigilância como Elemento de Desenvolvimento Local", que iniciou as atividades no ano de 2015 e propõe criar um processo dialógico referenciado nas ações de promoção, prevenção, redução de danos e agravos, permitindo assim o empoderamento da comunidade. O projeto de extensão envolveu 15 alunos de diferentes cursos de graduação do Instituto de Ciências Biológicas e da Saúde da Pontifícia Universidade Católica de Minas Gerais - PUC Minas, Brasil, em diversas práticas educativas na comunidade acadêmica da PUC Minas em Belo Horizonte e Betim, além dos habitantes locais. Abrangeu diferentes equipamentos sociais de saúde e de educação, tais como creches, escolas, instituição de longa permanência para idosos e grupos de convivência, para propiciar maior interação entre os discentes e a população através de ações quinzenais, seguindo as datas comemorativas referentes à prevenção de doenças, do calendário da saúde. Os acadêmicos foram capacitados pelos docentes do projeto para a realização das ações desenvolvidas em cada prática educativa.

Destaca-se que todas as práticas foram desenvolvidas em instituições parceiras, a partir da assinatura de termo de consentimento firmado entre a universidade e tais serviços, sendo todas as práticas planejadas e executadas a partir da demanda e da realidade de cada parceiro no projeto.

No presente artigo é relatada a experiência de quatro práticas educativas com foco nas condições crônicas de doenças cardiovasculares, hipertensão arterial, Diabetes Mellitus e obesidade, desenvolvidas por um grupo de sete extensionistas e duas docentes da PUC Minas, unidade Barreiro. Essas ações tiveram como objetivo principal oferecer ao público informações sobre a prevenção dessas doenças, aliadas a boa alimentação e ao autocuidado orientado, entendendo a educação em saúde numa vertente progressista e libertadora, para além da transmissão de conhecimentos. As práticas foram realizadas no hall do Restaurante Popular Dom Mauro Bastos, onde o público é composto principalmente por idosos, jovens estudantes da região e trabalhadores das empresas locais. Também foram realizadas ações no hall principal da PUC Minas Barreiro com professores, alunos e funcionários da unidade e, ainda, com alunos do $8^{\circ}$ ano de uma escola municipal, sendo as atividades realizadas na sala de aula da turma envolvida. Todos os locais são situados em Belo Horizonte - Minas Gerais e as práticas foram desenvolvidas nos meses de Abril, Outubro e Novembro de 2015, com consentimento e autorização de todos os locais, ou seja, as responsáveis técnicas do Restaurante Popular, a diretoria acadêmica da PUC Minas e a direção da escola e a coordenadora do Programa Saúde na Escola, respectivamente. 
Nas intervenções sobre "Hipertensão Arterial" e "Diabetes Mellitus", realizadas no Restaurante Popular Dom Mauro Bastos e na PUC Minas, as pessoas eram abordadas pelos extensionistas, individualmente, com uma breve conversa, para a discussão sobre os fatores de risco e a gravidade dessas doenças. Já na escola, as intervenções sobre a "Saúde do coração" e o "Combate à obesidade" as informações foram articuladas de forma dinâmica através de jogos, com o intuito de fazer os participantes se envolverem na discussão, considerando que o público era composto por adolescentes. Além disso, dicas e cuidados a serem tomados em relação à prevenção e ao tratamento dessas patologias também foram abordados em todas as práticas anteriormente citadas.

A ideia principal foi oferecer ao público informações sobre os riscos e as consequências da má alimentação associados a tais condições crônicas. Os extensionistas esclareceram dúvidas sobre as características das doenças e o que pode ser feito para se evitar e controlar as mesmas, além de fornecerem dicas nutricionais para amenizar os agravos, como, por exemplo, reduzir o consumo de sal, açúcar e óleo no preparo dos alimentos, temperos prontos, embutidos e demais alimentos industrializados ricos em sódio, açúcar e gordura. O intuito era que os participantes entendessem e discutissem as informações para fazerem escolhas mais conscientes dos alimentos a serem consumidos.

$\mathrm{Na}$ prática educativa "Combate à Hipertensão Arterial” foram distribuídas aos participantes amostras de "sal de ervas", com 50 gramas cada (Figura 1). Visando reduzir o consumo de sal, profissionais da área da saúde e órgãos governamentais brasileiros recomendam o uso de ervas frescas ou desidratadas, especiarias, limão e alho para temperar carnes, saladas e sopas. O "sal de ervas" auxilia na redução do sal em sua totalidade, agregando propriedades antioxidantes e anti-inflamatórias provenientes das ervas desidratadas (ARAÚJO, 2012). O mesmo foi preparado pelos extensionistas do projeto, e seus ingredientes foram: sal comum, noz moscada, hortelã, cebolinha e salsinha desidratadas. As amostras do "sal de ervas" acondicionadas em embalagens plásticas transparentes, e folder com a receita do preparo e algumas dicas foram oferecidos aos participantes. $\mathrm{O}$ folder educativo continha informações sobre a recomendação diária de consumo de sódio pela OMS e o teor deste nutriente em alguns alimentos industrializados (IGNÁGIO et al., 2013). Nesta prática foram apresentados também os kits de sal da Clínica de Nutrição da PUC Barreiro, que são compostos por pequenos vasilhames contendo a exata quantidade de sal que determinado alimento possui. Os kits foram utilizados como forma de conscientização em relação ao consumo excessivo de sal, uma vez que possibilitam melhor visualização da quantidade de sal presente nos alimentos usualmente ingeridos pela população (Figura 1). 


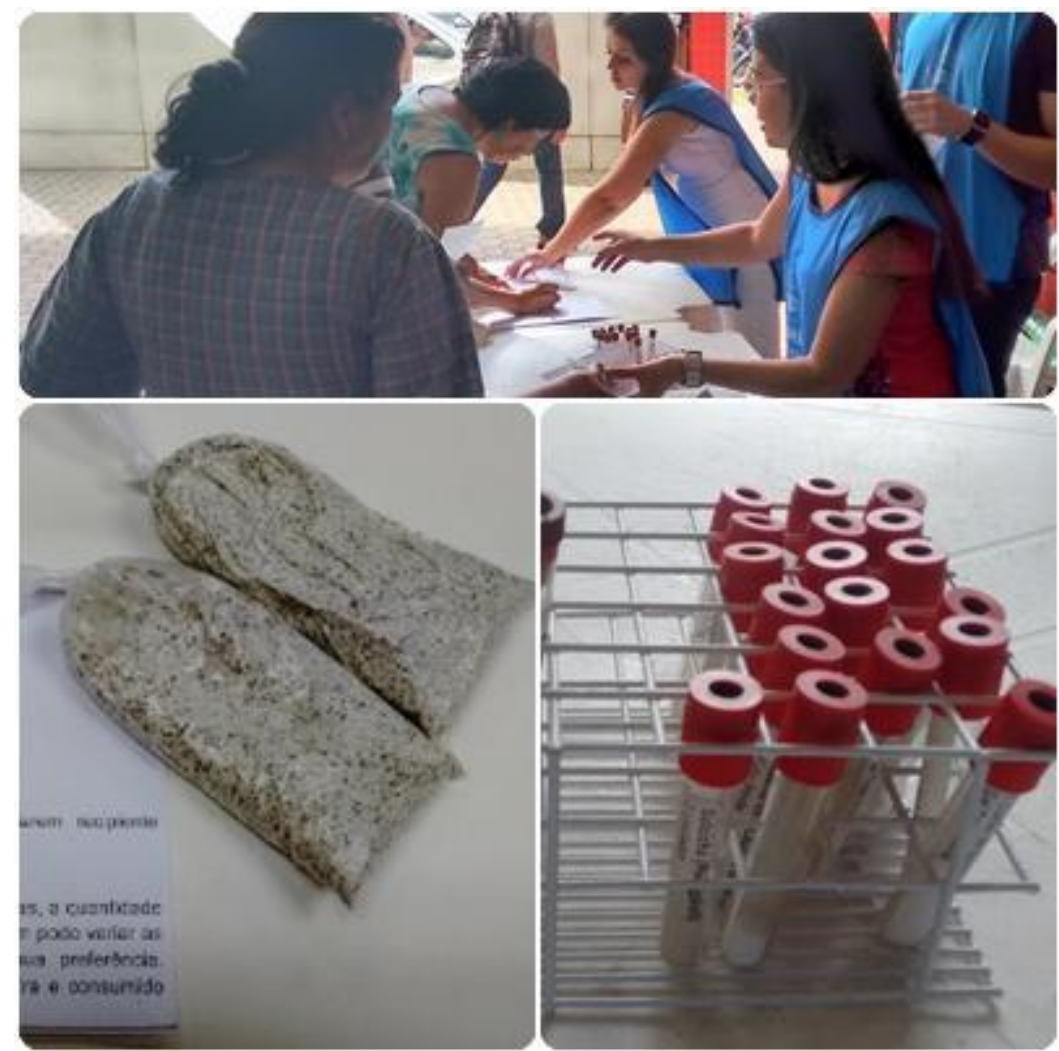

Figura 1 - Prática educativa sobre Hipertensão Arterial, "Sal de ervas” e kits de sal, Restaurante Popular Dom Mauro Bastos, Belo Horizonte - Minas Gerais.

Fonte: Elaborado pelas autoras, 2015.

$\mathrm{Na}$ ação em comemoração ao "Dia Mundial de Combate à Obesidade" foi realizado um jogo de perguntas e respostas com alunos do $8^{\circ}$ ano da escola participante, a fim de gerar conhecimento sobre as ações preventivas e de tratamento para a obesidade. Os participantes foram divididos em dois times para responder perguntas relacionadas ao tema e, a cada acerto, os pontos foram computados, sendo a equipe vencedora aquela que obteve maior número de acertos.

Na prática "Dia Mundial do Coração" também foi realizado um jogo com os mesmos alunos do $8^{\circ}$ ano com o intuito de dar continuidade às ideias propostas na prática anterior, e assim, vincular os dois temas para a promoção da saúde. O jogo foi realizado em forma de "batata quente" com perguntas sobre o tema, que foi explicado previamente em uma roda de conversa. Para realizar o jogo as músicas eram escolhidas pelos alunos e apresentada com violino, como forma de despertar maior atenção dos participantes. No decorrer da prática foi possível transmitir conhecimento aos adolescentes quanto aos fatores de risco para o desenvolvimento de doenças cardiovasculares e informar quanto ao seu tratamento, alertando sobre os hábitos alimentares saudáveis, prática de atividade física e rotina familiar. 
$\mathrm{Na}$ intervenção com o tema "Combate ao Diabetes" foi distribuído em copo descartável de $50 \mathrm{~mL}$ maçã em calda, doce preparado sem a adição de açúcar. A proposta era desmistificar o uso do açúcar comum de cozinha como ingrediente essencial para se fazer uma sobremesa apetitosa. Além da receita da maçã em calda, foram impressas outras receitas como strogonoff de chocolate, sorvete de gelatina e bolo de laranja, que foram distribuídas ao público geral participante da prática no Restaurante Popular (Figura 2).
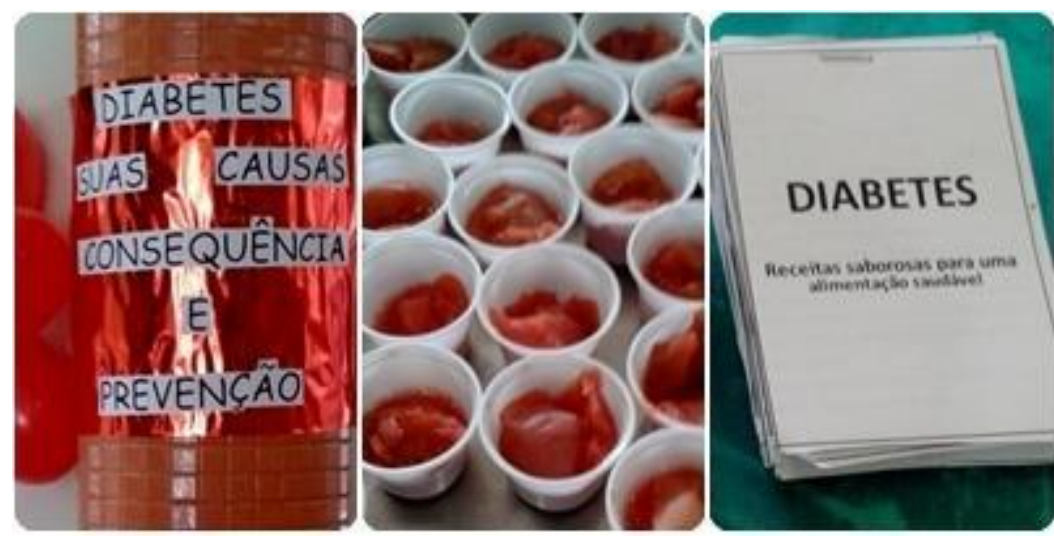

Figura 2 - Prática educativa sobre Diabetes, maçã em calda, receitas para diabéticos, Restaurante Popular Dom Mauro Bastos, Belo Horizonte - Minas Gerais. Fonte: Elaborado pelas autoras, 2015.

\section{RESULTADOS E DISCUSSÃO}

Os resultados obtidos em cada prática foram satisfatórios, pois houve a troca de conhecimentos, beneficiando tanto os extensionistas quanto aos participantes. Houve crescimento da capacidade dos alunos que desenvolveram as práticas como agentes de transformação social, já que, acredita-se que eles contribuíram para ampliar o conhecimento da população local de forma original e criativa. Além disso, o conhecimento e a prática de técnicas realizadas pelos discentes e docentes com o objetivo de se alcançar crescimento profissional e pessoal foram ampliados, pois com o contato e o diálogo há maior sensibilidade em relação aos participantes. Deucher, Buzzello e Zampieri (2004, p.9) consideram que a extensão tem importância na sociedade e comunidade acadêmica, uma vez que promove interação entre ambas às partes e "estabelece canais de comunicação, articula ensino e pesquisa, aproxima o acadêmico e o popular, proporcionando a criação de novos saberes, produzindo relações transformadoras entre a universidade e a sociedade".

A prática "Combate à Hipertensão Arterial" teve duração de 3 horas em cada local e participaram 355 pessoas no Restaurante Popular e 101 na PUC Barreiro, totalizando 456 
participantes. Pode-se aferir que houve boa aceitação da intervenção realizada a partir da troca de experiências no momento da ação e dos relatos dos participantes ao se depararem com o tema e todos os benefícios apresentados, pois apesar da hipertensão arterial ser um problema muito comum no Brasil, algumas pessoas ainda não sabem como preveni-la de forma simples. Além disso, várias pessoas hipertensas desconhecessem as alternativas para melhorar seu estado de saúde. Para tanto, nem sempre é necessário recorrer somente aos medicamentos, sendo que estilos de vida mais saudáveis podem resultar em melhorias nas condições de saúde. Sendo assim, houve interesse das pessoas que participaram da prática educativa sobre formas de prevenção das doenças crônicas, ação fundamental considerando a situação epidemiológica brasileira.

O "sal de ervas" despertou a curiosidade da maioria das pessoas pelo aroma, aparência e/ou porque não conheciam ou usavam temperos artificiais substitutivos ao sal. A ideia de que a receita é adaptável em relação aos ingredientes utilizados manifestou interesse aos participantes, já que isso permite o uso de outras ervas às usadas na amostra oferecida. Com a utilização de preparações substitutas a alimentos que não devem ser ingeridos em excesso, há maior incentivo para que as pessoas mudem seus hábitos alimentares. Assim é implementada a educação em saúde, como um processo contínuo de formação que no início gera estranhamento, mas que acaba por se tornar hábito (CECCIM; FERLA, 2008).

A exposição dos kits de sal da Clínica de Nutrição da PUC Barreiro durante a prática educativa foi impactante, pois as pessoas de fato não sabiam a quantidade de sal que muitos alimentos usualmente consumidos contêm. A comparação da quantidade de sal de alguns alimentos com a recomendação diária estabelecida para a população brasileira foi outro aspecto positivo que os kits de sal possibilitaram, pois dessa forma as pessoas visualizaram que muitos alimentos consumidos diariamente possuem grande quantidade de sódio o que ao longo da vida podem acarretar no surgimento de DCNT, como a hipertensão arterial (MENDES, MORAES, GOMES, 2014). Sichieri e Cardoso (2016) reforçam que o crescente consumo de sal aumenta a prevalência global de hipertensão, principalmente entre os jovens que possuem alto consumo de alimentos ultraprocessados.

Um aspecto negativo da intervenção foi à desistência de algumas pessoas em participar ao perceberem que não se tratava de uma atividade que envolvesse também a aferição de pressão arterial que é uma prática comum em ações que abordam o tema hipertensão. No entanto, sabese que a mudança do estilo de vida é uma das principais formas de prevenção da hipertensão arterial e até mesmo de tratamento, bem como a adequação nos hábitos alimentares, principalmente quanto à ingestão de sódio. Com base nesta experiência observou-se que ainda há necessidade de mais intervenções educativas que possam contribuir para o empoderamento da 
população perante as DCNT, como a hipertensão, que auxiliem na resolução de inúmeras dúvidas que muitas vezes não são sanadas apenas em consultas médicas.

Ressalta-se a importância do trabalho de promoção e prevenção de doenças que abranjam toda a população, como estímulo ao autocuidado e possibilidade de contribuir para melhora dos hábitos alimentares e qualidade de vida. Deste modo, a extensão universitária tem um papel importante no incentivo à atenção da saúde, sendo necessário para isso, que a própria comunidade procure perceber a universidade como um espaço para reflexão e aprendizado, e “adentrar ao seu interior" para que haja aproximação entre as partes e maior efetividade das ações propostas (RODRIGUES, 2006).

Em relação a prática "Combate ao Diabetes", com duração de 3 horas, o número de pessoas abordadas foi de 195 no Restaurante Popular. Nessa prática muitas pessoas ficaram interessadas na degustação da maçã em calda, que foi aceita por todas as faixas etárias, o que pôde potencializar a proposta inicial da ação. Os participantes também tiveram interesse nas receitas impressas, muitas vezes levando mais de uma unidade do folheto consigo. Além disso, o fato de haver outras receitas, além da exposta para degustação, chamou atenção dos usuários do restaurante e a maioria afirmou que utilizaria alguma das preparações. Com isso, as receitas impressas mostraram-se eficientes em relação ao objetivo inicial que era de conhecimento de formas de substituição das sobremesas tradicionais que levam grande quantidade de açúcar, podendo resultar ainda na replicação das mesmas pelos participantes nas suas residências.

Assim como a intervenção anterior, o aspecto negativo foi o desinteresse de pessoas que queriam apenas fazer o controle da glicemia, e como essa atividade não foi realizada, muitas deixaram de participar da prática educativa. Ao contrário da prática de combate à hipertensão arterial em que mesmo as pessoas sem a doença participaram, houve resistência por parte das que não eram portadoras do Diabetes Mellitus. Entretanto, em ambas as práticas, muitas pessoas ao serem questionadas se eram portadores da patologia, não sabiam responder. Essa situação pode ser explicada pelo fato de que a maioria se interessa pelo seu estado de saúde atual, porém não se sentem atraídas por informações que têm como objetivo prevenir doenças. O autocuidado é de grande importância para a qualidade de vida, mas, deve-se considerar que a cultura hegemônica da população é de cuidado à saúde nos processos de adoecimento, ou seja, espera-se a doença aparecer para então tratar, o que normalmente se relaciona à falta de tempo vivida atualmente pela correria do dia-a-dia e/ou pela cultura formada na população em que o foco é tratar a doença, e não preveni-la com exames básicos de rotina e acompanhamento de profissionais para manutenção da saúde (BARROS, 2002; SOUZA et at., 2005). É preciso formação ampliada na educação em saúde, em que as pessoas consigam perceber e se conscientizar que cuidar da saúde, 
e não da doença, é a melhor forma de se viver com qualidade, considerando as possibilidades e os recursos possíveis de cada contexto. Xavier, Brittar e Ataíde (2009) reforçam que o autocuidado é importante e gerador de menos complicações à saúde no decorrer da vida. Já Bento, Mariano e Andrade (2010) afirmam que os profissionais de saúde devem deter o conhecimento dessa prática para articular com a população, que muitas vezes não têm acesso, para que cada indivíduo possa cuidar de si mesmo. Romper com o paradigma da saúde como ausência de doença deve fazer parte do cotidiano da formação e da prática em saúde, com ações que explicitem a responsabilidade de cada sujeito pela sua condição, na busca por uma cultura promotora da saúde. Apesar do menor número de participantes, também foi uma prática educativa com resultados positivos e interesse de quem participou, considerando o envolvimento e a aprovação de todos em relação ao sabor da receita.

Santos e Castro (2014) ao tentarem colaborar com mudanças no estilo de vida de uma comunidade de Petrolina-Pernambuco, promoveram uma série de atividades de incentivo a alimentação saudável e a prática regular de atividades físicas, através de dinâmicas, experiências práticas e rodas de conversa. Os autores observaram que a comunidade apresenta muitos questionamentos sobre hábitos de vida saudáveis e a forma como estes podem ser praticados no dia a dia. Nesta prática realizada no nordeste brasileiro, nutricionistas puderam discutir com a comunidade formas de se controlar e prevenir agravos associados à hipertensão e ao diabetes. Santos e Castro (2014) ainda relataram que os participantes da comunidade reconheceram a importância de eventos como o ocorrido para que o acesso a informações desse tipo seja democratizado e chegue até as comunidades onde as pessoas realmente necessitam de tais orientações.

Na prática sobre "Combate à Obesidade", com duração de 1 hora, houve a participação de 32 alunos do $8^{\circ}$ ano de uma escola municipal. O conteúdo discutido com os alunos foi assimilado e os mesmos demonstraram satisfação e entusiasmo durante a intervenção, principalmente porque o tema abordou a estética corporal. Este é um assunto que desperta interesse ao público jovem, tendo em vista que na atualidade entende-se como corpo ideal um modelo de corpo esguio e atlético, levando o adolescente a querer se aproximar deste padrão, pois muitas vezes, quem se encontra fora dele é visto de forma negativa (AMARAL, GALEGO e NOVELLO, 2016; RIBEIRO, 2016). Um aspecto negativo a ser destacado é que a ação ocorreu depois do horário do recreio, sendo assim, os alunos chegaram eufóricos e inicialmente houve dificuldade em conseguir a atenção dos mesmos. Apesar disso, durante a ação os alunos foram se interessando e assim, a prática obteve sucesso. Santos et al. (2014, p. 21) enfatizam que se “conseguir canalizar adequadamente o potencial crítico, criativo, inovador e participativo do 
adolescente, conseguirá, além de envolvê-lo nas práticas de educação em saúde, mudanças positivas na vida desse sujeito".

Na prática com tema "Dia Mundial do Coração" participaram 30 alunos da mesma turma, ou seja, faltaram neste dia dois alunos. Os participantes se mostraram interessados sobre o tema, a curiosidade dos alunos fez com que a prática se tornasse dinâmica e, assim, os extensionistas puderam sanar dúvidas comuns aos alunos em relação ao tema proposto, como por exemplo, os valores de referência para considerar um indivíduo hipertenso, como a aterosclerose se desenvolve e suas consequências ao organismo, diferenças entre infarto e acidente vascular encefálico, quais alimentos devem ser evitados, entre outros.

Santos et al. (2005) ao realizarem práticas de educação nutricional com escolares do ensino fundamental de 16 instituições públicas e privadas de Florianópolis, Santa Catarina, observaram que é necessário encontrar estratégias educativas que perpassem a simples assimilação do saber, adentrando na tão esperada mudança de comportamento. Os métodos utilizados nas abordagens educativas devem contribuir para o desencadeamento de decisões e ações que levem à transformação dos hábitos alimentares. Além disso, é preciso vincular as atividades educativas aos conteúdos vistos nas disciplinas escolares e a práticas de atividades físicas, para que se consiga estabelecer hábitos alimentares e de estilo de vida mais saudáveis. Além disso, as atividades educativas desenvolvidas com crianças e adolescentes devem ser atrativas para esse público, com linguagem acessível e estratégias criativas de modo a facilitar o desenvolvimento das atividades (SANTOS et al., 2014).

Sabe-se que os materiais didáticos podem ser importantes para enriquecer as práticas educativas, mas não são essenciais, uma vez que, o desempenho da ação educativa está diretamente relacionado com a qualificação das informações, a participação e envolvimento dos sujeitos. Essa reflexão foi fundamental para o reconhecimento de que a educação em saúde pode ser feita a partir do encontro de pessoas que tem conhecimentos diferentes e que, por meio de prática dialógica podem reconhecer potenciais de melhoria para o cuidado com a sua saúde, desmistificando a prática educativa hegemônica feita por meio de palestras, uso de recursos audiovisuais, procedimentos, brindes e com pouco diálogo.

$\mathrm{Na}$ extensão percebe-se nitidamente que sua função é tornar a universidade "locus" distinto de conhecimento e saber, que intercede com a sociedade, arquitetando saberes variados e inovadores, mediando dessa forma o conhecimento da população com o conhecimento científico presente na Universidade (OLIVEIRA, 2004).

As ações educativas em saúde são um trabalho coletivo e que devem ser incentivadas com o objetivo de levar informações aos participantes e contribuir com a promoção da saúde. A 
A prática educativa na prevenção de condições crônicas

extensão universitária é uma forma de ajudar nesse sentido, pois além de levar informações à comunidade, trás conhecimentos práticos para os alunos, sendo válido para ambos os lados.

\section{CONSIDERAÇÕES FINAIS}

Com a realização das práticas educativas pôde-se concluir que, apesar de a tecnologia estar cada vez mais avançada, com várias ferramentas de pesquisa disponíveis possibilitando informação e conhecimento, muitas pessoas não têm informações básicas referentes ao cuidado com a saúde e a prevenção de doenças. Além disso, transformar o volume de informações de saúde disponíveis por diferentes fontes em ações de cuidado requer mudanças nas estratégias educativas para o empoderamento da população, sendo possível por meio de práticas cada vez mais dialógicas.

O conhecimento científico adquirido na comunidade acadêmica pelos discentes extensionistas foi de fundamental importância para a realização das intervenções, já que houve participação, e consequentemente, muitas perguntas. Por outro lado, pelo potencial de "ensinagem" em que as trocas permanentes entre quem ensina e quem aprende, é que a extensão universitária foi benéfica não somente aos participantes das intervenções, mas também aos extensionistas, uma vez que possibilitou conhecimento além das teorias aprendidas na sala de aula.

Observou-se na execução do projeto "Retratos do Cotidiano em Saúde: A Vigilância como Elemento de Desenvolvimento Local" que as atividades realizadas de forma pontual em diferentes instituições parcerias não foram satisfatórias no sentido do público não ter atividades contínuas relacionadas à educação em saúde. Desta forma, observou-se a importância da continuidade das práticas educativas para que a prevenção possa ser alcançada por meio da promoção em saúde, pois assim as pessoas poderão prevenir-se buscando estilos de vida mais saudáveis.

O desafio para os profissionais de saúde na atualidade está em motivar as pessoas que já possuem conhecimento sobre manutenção da saúde, mas que não praticam as ações cuidadoras. A extensão dentro da universidade tem papel fundamental no aspecto de promoção e prevenção da saúde, pois através dela leva-se o conhecimento às pessoas e há troca de saberes entre acadêmicos e comunidade, ampliando a visão e o conceito de saúde do aluno e aproximando-o dos problemas recorrentes em determinado local, gerando maior envolvimento social dos mesmos. Assim, a prática extensionista é uma forma de, aos poucos, promover educação em 
A prática educativa na prevenção de condições crônicas

saúde e, consequentemente, melhorar a qualidade de vida da comunidade participante. Além disso, o contato com as pessoas torna os educandos cada vez mais experientes, favorecendo a formação de profissionais de qualidade com maior valor de ética e humanismo.

\section{REFERÊNCIAS}

AMARAL, Aline Carla Chagas do; GALEGO, Beatriz Valle; NOVELLO, Daiana. Estado nutricional e percepção corporal entre adolescentes de uma escola do município de Guarapuava, PR. Revista da Universidade Vale do Rio Verde, Três Corações, v. 14, n. 1, p. 383-392, 2016.

ARAÚJO, Íris Braz da Silva. Otimização do uso de "sal de ervas" e cloreto de potássio na substituição parcial do cloreto de sódio em corte e em linguiça de frango. Dissertação (Mestrado) - UFBP/CCHSA. Bananeiras: [s.n], 2012.

BARROS, José Augusto C. Pensando o processo saúde doença: a que responde o modelo biomédico? Rev. Saúde e Sociedade. v. 11. p. 67-84. 2002.

BENTO, Deonízio Gercy; MARIANO, Ana Flávia; ANDRADE, Selma Regina de. Autocuidado e recursos assistenciais aos portadores de hepatite B. Ciênc. Cuid. Saúde, v. 9, n. 2, p. 285-291. 2010.

DEUCHER, Carolina Vieira; BUZZELLO, Cristiane dos Santos; ZAMPIERI, Maria de Fátima Mota. Grupo de gestante e/ou casais de grávidos: a universidade interagindo com a comunidade. Extensio: R. Eletr. de Extensão, n. 1, p. 1-10, 2004.

CECCIM, Ricardo Burg; FERLA, Alcindo Antônio. Educação e saúde: Ensino e cidadania como travessia de fronteiras. Trab. Educ. Saúde, v. 6, n. 3, p. 443-456. 2008.

CORRÊA, Priscila Milano; HENKES, Stela; LANAU, Renan; DUTRA, Camila Marins; RIBEIRO, Cristina Dutra; FRANCO, Ozeia Simões et al. Vivências em promoção da saúde: articulando saberes com estudantes de escolas públicas. Entensio: R. Eletr. de Extensão, v.12, n.19, p.14-24, 2015.

DIVINO, Anne Emiler do Amo; COSTA, Carmen Lucia Neves do Amaral; OLIVEIRA, Carla Eduarda Luz de; COSTA, Christian Alexandra de Carvalho; SOUZA NETA, Hilda Rollemberg de; CAMPOS, Lucir da Silva; MENEZES, Raira Mota de Jesus; CABRAL, Stephanie Costa da Silva. A extensão universitária quebrando barreiras. Cadernos de Graduação: Ciências Humanas e Sociais (UNIT), v. 1, n. 16, p. 135-140, 2013.

DUNCAN, Bruce Bartholow; CHOR, Dóra; AQUINO, Estela M.L.; BENSENOR, Isabela M.; MILL, José Geraldo; SCHMIDT, Maria Inês; LOTUFO, Paulo Andrade; VIGO, Álvaro; BARRETO, Sandhi Maria. Doenças crônicas não transmissíveis no Brasil: prioridade para enfrentamento e investigação. Revista de Saúde Pública, v. 46, p. 126-134. 2012.

FIGUEIREDO, Maria Fernanda Santos; RODRIGUES-NETO, João Felício; LEITE, Maísa Tavares Souza. Modelos aplicados às atividades de educação em saúde. Rev. Bras. Enferm., v. 63, n. 1, p. 117-121. 2010. 
IGNÁGIO, Ana Caroline Ferreira; RODRIGUES, José, Tarcisio de Domenico; NIIZU, Patricia, Yuasa; CHANG, Yoon, Kil. Efeito da Substituição de Cloreto de Sódio por Cloreto de Potássio em Pão Francês.

Braz. J. Food Technol., v. 16, n. 1, p. 1-11, 2013.

MALTA, Deborah Carvalho; MORAIS NETO, Otaliba Libânio de; SILVA JUNIOR, Jarbas Barbosa. Apresentação do plano de ações estratégicas para o enfrentamento das doenças crônicas não transmissíveis no Brasil, 2011 a 2022. Epidemiol. Serv. Saúde, v. 20, n. 4, p. 425-438, 2011. MANSO, Maria Elisa Gonzalez; CÂMARA, Rafaela; SOUZA, Suely Alves; MACIEL, Talita Dias; FARINA, Danielle Baptista Livrari. Programa de gerenciamento de doenças crônicas me um plano de saúde, São Paulo, Brasil. Cienc. Cuid. Saude, v. 15, n. 2, p. 321-327, 2016.

MELLO, Ana Paula Abraão; BELO, Lorrany Aparecida de Oliveira; PONTES, Angelita Evaristo Barbosa; PAGOTTO, Valéria; NAKATANI, Adélia Yaeko Kyosen; MARTINS, Karine Anusca. Estudo de base populacional sobre excesso de peso e diabetes mellitus em idosos na região metropolitana de Goiânia, Goiás. Geriatr. Gerontol. Aging., v. 10, n. 3, p. 151-7. 2016.

MENDES Gisele Soares; MORAES Clayton Franco; GOMES, Lucy. Prevalência de hipertensão arterial sistêmica em idosos no Brasil entre 2006 e 2010. Rev. Bras. Med. Fam. Comunidade, v. 9, n. 32, p. 273-278, 2014.

MENDES, Eugênio Vilaça. $\mathbf{O}$ cuidado das condições crônicas na atenção primária à saúde: O imperativo da consolidação da estratégia da saúde da família. Brasília: Organização PanAmericana da Saúde, 2012. 512p.

ORGANIZAÇÃO MUNDIAL DA SAÚDE. Global status report on noncommunicable diseases. 2014. Disponível em: <http://www.who.int/campaigns/world-health-day/2013/es/>. Acesso em: 18. mai. 2015.

PORTAL BRASIL - Ministério da Saúde. Política Nacional de Saúde/ Doenças crônicas não transmissíveis. Ministério da Saúde, 2014. Disponível em: <http://www.brasil.gov.br/saude/2014/12/pesquisa-revela-que-57-4-milhoes-de-brasileirostem-doenca-cronica>. Acesso em 3. mar. 2016.

RIBEIRO, Karla Carolina Silveira; SANTOS, Marcela Silva dos; SILVA, Josevânia da, PICHELLI, Ana Alayde Werba Saldanha. A (in)satisfação com o corpo e a vulnerabilidade aos transtornos alimentares em adolescentes. Cad. Ed. Tec. Soc., Inhumas, v. 9, n. 2, p. 194-203, 2016.

RODRIGUES, Rogério. A extensão universitária como uma práxis. Em Extensão, v. 5, p. 8488 , p. 84-88, 2006.

SANTOS, Alesson Rodrigo Santos e; CASTRO, Iago Alves de. Promoção da saúde por meio de orientação nutricional e atividades físicas com enfoque na hipertensão arterial sistêmica e diabetes mellitus no bairro José e Maria. Extramuros: Rev. Ext. da Univasf, v. 2, n. 2, p. 11-17, 2014.

SANTOS, Cristhiane Schneider; GABRIEL, Cristine Garcia ; ROSSI, Camila Elizandra; ANTONELLI, Etienne Jacques; ALVES, Diana de Oliveira; FAVARIN, Tatiane; Schmitt, Sabrina dos Santos. Ações educativas em nutrição para prevenção de obesidade em escolares de Florianópolis-SC. Extensio: R. Eletr. de Extensão, n. 2, p. 1-19, 2005.

SANTOS, Jaqueline Silva; ANDRADE, Raquel Dully; MELLO, Débora Falleiros de; MAIA, 
Maria Ambrosina Cardoso. Educação em saúde na adolescência: contribuições da Estratégia Saúde da Família. Rev. Soc. Bras. Enferm. Ped., v. 14, n. 1, p. 20-26. 2014.

SCHMIDT, Maria Inês; DUNCAN, Bruce Bartholow; SILVA, Gulnar Azevedo e; MENEZES, Ana Maria; MONTEIRO, Carlos Augusto; BARRETO, Sandhi Maria; CHOR, Dora; MENEZES, Paulo Rossi. Doenças crônicas não transmissíveis no Brasil: carga e desafios atuais. Séries Saúde no Brasil 4, p. 61-74, 2011.

OLIVEIRA, Claudia Hochheim. Qual é o Papel da Extensão Universitária? Algumas Reflexões Acerca da Relação entre Universidade, Políticas Públicas e Sociedade. In: CONGRESSO BRASILEIRO DE EXTENSÃO UNIVERSITÁRIA, 2., 2004, Belo Horizonte. Anais... - 12 a 15 de setembro de 2004. Disponível em: https://www.ufmg.br/congrext/Gestao/Gestao15.pdf. Acesso em: em 9. nov. 2016.

SICHIERI, Rosely; CARDOSO, Marly A. ERICA: estudo dos riscos cardiovasculares em adolescentes. Rev. Saúde Públ., v. 50, suppl 1, 1S-2S, 2016.

SOUZA, Aline de Corrêa; COLOMÉ, Isabel Cristina dos Santos; COSTA, Lilian Escopelles Deves; OLIVEIRA, Dora Lúcia Leidens Corrêa de. A educação em saúde com grupos na comunidade: uma estratégia facilitadora da promoção da saúde. Rev. Gaúcha de Enfer., v. 26, n. 2, p. 147-153, 2005.

VILLELA, Lenice de Castro Mendes; GOMES Fábio Eduardo; MELÉNDEZ, Jorge Gustavo Velásquez. Tendência da mortalidade por doenças cardiovasculares, isquêmicas do coração e cerebrovasculares. Rev enferm UFPE, v. 8, n. 9, p.3134-41, 2014.

XAVIER, Antônia Tayana da Franca; BITTAR, Daniela Borges; ATAÍDE, Márcia Barroso Camilo de. Crenças no autocuidado em diabetes - implicações para a prática. Texto Contexto Enferm., v. 18, n. 1, p. 124-30. 2009. 